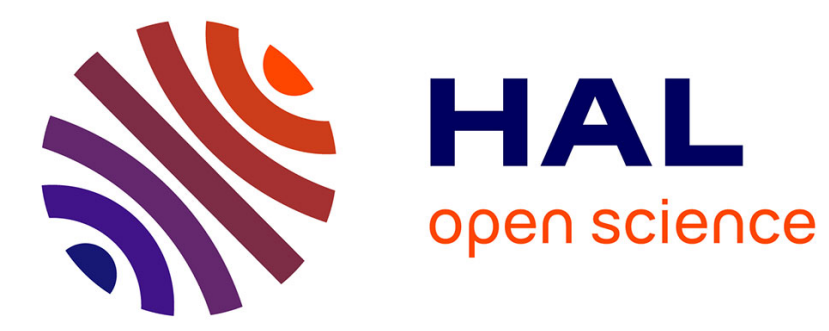

\title{
Des exégètes du XVIe siècle Face à la conversion et à l'apostasie
}

Thierry Wanegffelen

\section{To cite this version:}

Thierry Wanegffelen. Des exégètes du XVIe siècle Face à la conversion et à l'apostasie. Revue de l'histoire des religions, 1993, 210, pp.413-430. hal-00294487

\section{HAL Id: hal-00294487 \\ https://hal.science/hal-00294487}

Submitted on 9 Jul 2008

HAL is a multi-disciplinary open access archive for the deposit and dissemination of scientific research documents, whether they are published or not. The documents may come from teaching and research institutions in France or abroad, or from public or private research centers.
L'archive ouverte pluridisciplinaire HAL, est destinée au dépôt et à la diffusion de documents scientifiques de niveau recherche, publiés ou non, émanant des établissements d'enseignement et de recherche français ou étrangers, des laboratoires publics ou privés. 


\title{
Des exégètes du XVIe siècle Face à la conversion et à l'apostasie
}

\author{
par Thierry Wanegffelen \\ Centre de Recherches d'Histoire moderne \\ Université de Paris I (Panthéon-Sorbonne)
}

La Réforme du XVIe siècle s'est distinguée de la Contre Réforme notamment en proposant de « relectures ${ }^{1}$ » originales des grands textes scripturaires. Le fait est admis et il suffit pour s'en convaincre de songer au rôle assigné unanimement par l'historiographie au commentaire de Martin Luther sur l'Épître aux Romains sur l'itinéraire spirituel du Réformateur et en conséquence sur l'histoire religieuse de l'Empire puis de l'Europe. L' « explosion libératrice » - l'expression est de Pierre Chaunu ${ }^{2}$ - résulte d'une véritable expérience de « relecture ».

De tels phénomènes de « relecture » ont été bien étudiés et il n’en sera pas question ici. Le dossier proposé porte sur de tout autre passages scripturaires, moins fondamentaux, bien sûr, mais c'est en cela qu'ils peuvent intéresser. L'idée est en effet de savoir si l'apparition et la constitution de confessions chrétiennes rivales à partir de 1520 et tout au long du XVIe siècle ont ou non déterminé un changement dans le discours exégétique global catholique et protestant. Autrement dit, y a-t-il eu production de discours confessionnels distincts ou, pour le moins, « confessionalisation » du discours, c'est-à-dire prise en compte par les exégètes de tous bords de la nouvelle situation ecclésiologique ?

\footnotetext{
${ }^{1}$ Le phénomène des «relectures » successives et incessantes, en fait, des textes bibliques a été mis en lumière par les exégètes contemporains, notamment Pierre Gibert, dont on peut consulter La Bible à la naissance de l'Histoire, Paris, 1979. Un exemple de relecture dans la Bible est fourni par l'un des épisodes les plus fameux du livre de l'Exode : l'histoire du Veau d'Or; il est exposé dans Thierry Wanegffelen, «L’historien et la Création », Études, 375, 1991, p. 465-473, en particulier p. 468-469. On voit ce que c'est que « relire » pour les exégètes actuels : c'est pendre le même texte, pour lui faire die quelque chose de nouveau, en rapport avec la situation présente.

${ }^{2}$ Pierre Chaunu, Le Temps des Réformes. Histoire religieuse et système de civilisation. La crise de la Chrétienté. L'éclatement (1250-1550), Paris, 1975, p. 388.
} 
L'étude a un enjeu historiographique que vient de révéler l'emploi de ce terme de « confessionalisation ». Une bonne part du travail actuel des historiens du fait religieux en France aux XVIe et XVIIe siècles paraît être en effet l'adaptation au cas français de deux concepts (souvent confondus à tort), forgés par les historiens allemands et propres à la situation ecclésiologique et socioculturelle de l'espace germanique à l'époque moderne ${ }^{3}$. Il s'agit de la Konfessionsbildung (qu'il faudrait peut-être rendre par «construction confessionnelle » et non par «confessionalisation » comme on le fait volontiers) et de la Konfessionalisierung (impossible à traduire sinon par « confessionalisation»); elles ne sont pas interchangeables. La «construction confessionnelle » prétend rendre compte d'une situation dans laquelle on voit se fonder et se mettre en place des «Églises » (ou « confessions ») chrétiennes rivales, au sein desquelles se développent deux manières de vivre le christianisme si distinctes l'une de l'autre qu'on serait presque tenté de les définir comme des « religions » différentes ${ }^{4}$; le concept entend même aller plus loin et faire de ces « Églises » le lieu de « civilisations » différentes.

Le concept de «confessionalisation» proprement dit pointe un autre aspect des choses : cette seconde voie a été frayée par les travaux indépendants l'un de l'autre, dans les années 1970 et 1980, de Wolfgang Reinhard et de Heinz Schilling, historiens respectivement du catholicisme et du

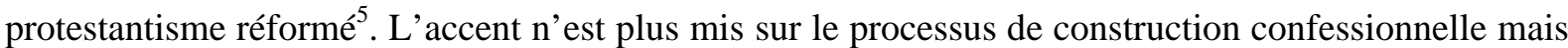
sur l'évolution qu'on croit percevoir dans l'espace allemand au sein des confessions. On est parti de

${ }^{3}$ Mise en place de la question dans Thierry Wanegffelen, « Les chrétiens face aux Églises en Europe aux XVIe et XVIIe siècles », Nouvelle Revue du Seizième Siècle, 11, 1993, p. 37-53.

${ }^{4}$ Ce concept de Konfessionsbildung a notamment été élaboré dans les années 1960 par Ernst Walter Zeeden : à partir des années 1560 on assiste à la mise en place des trois ensembles confessionnels rivaux, luthérien, réformé, catholique ; tout les oppose, mais leur construction se fait par des moyens assez semblables, dans la mesure où il s'agit partout d'imposer des modes de vivre collectifs pour consolider l'identité confessionnelle. Dans cette ligne historiographique s'inscrivent les travaux récents sur le protestantisme réformé de Heiko A. Oberman, « Eine Epoche. Drei Reformationen », dans Die Remormation. Von Wittenberg nach Gent, Göttingen, 1986, p. 283-299.

${ }^{5}$ On pourra lire deux recueils, publiés sous la direction de Wolfgang Reinhard, Bekenntnis und Geschichte : die Confessio Augustana im historischen Zusammenhang, Ringvorlesung der Universität Augsburg im Jubiläumsjahr 1980, Munich, 1981, et Humanismus des 15. und 16. Jahrhunderts, Weinsheim, 1984, ainsi que la thèse d'habilitation (1977-1978) de Heinz Schilling, Konfessionskonflikt und Staatsbildung : eine Fallstudie über das Verhällnis von religiösem und sozialem Wandel in der Frühneuzeit, am Beispiel der Grafsschaft Lippe, Gütersloh, 1981 ; pour saisir le concept allemand proprement dit de confessionalisation (Konfessionalisierung) s'impose toutefois la lecture des actes du Symposium de 1985 du Verein für Reformationsgeschichte, publié sous la direction de Heinz Schilling, Die reformierte Konfessionalisierung in Deutschland. Das Problem der «Zweiten Reformation», Wissenschaftliches Symposion des Vereins für Reformationsgeschichte, 1985, Gütersloh, 1986, et notamment l'article de H. Schilling, «Die "Zweite Reformation” als Kategorie der Geschichtswissenschaft », p. 387-437. 
l'étude du Palatinat allemand. De là a été dégagé le modèle suivant : à partir des années 1560, en Europe, le mouvement religieux ne serait plus venu d'en bas ; désormais, il aurait été contrôlé par en haut, princes et élites cultivées auraient utilisé le phénomène de construction d’Églises confessionnelles bien organisées pour affermir leur pouvoir politique et leur emprise intellectuelle.

Le dossier ici présenté est un apport à ce travail d'adaptation. E cas de l'exégèse permet de voir si la « construction confessionnelle » a été ou non effective, sinon totale, en France au cous du XVIe siècle. Une première difficulté a consisté à déterminer le corpus scripturaire à envisager. Je l'ai dit, des textes au cœur des débats entre confessions, comme l’Épître aux Romains, n’auraient rien apporté à la démonstration ; les textes retenus devaient pourtant permettre à leurs commentateurs d'exprimer une éventuelle situations de Konfessionsbildung. Intéressé par les questions d'apostasie, j'ai pu constater qu'il n'est pas d'occasion mieux venue pour dire son sentiment d'appartenance confessionnelle que lorsqu'on loue celui qui se convertit à sa confession ou vilipende celui qui la quitte, autrement dit l'apostat. Le fai est fréquent dans les textes du XVIe siècle et s'accompagne toujours de références à des épisodes bibliques, surtout, mais non exclusivement, évangéliques. Le Traité de l'Apostasie du pasteur réformé français Jean de L’Espine, qui date de 1582, fournit une liste assez complète des passages bibliques qui disent l'apostasie, le reniement de Dieu. Le chapitre III s'intitule «Dieu se départ des Apostats "; ces derniers y sont successivement comparés à Adam et Eve, au roi David, au fils prodigue, enfin à Pierre. Les passages vétéro-testamentaires évoqués par L'Espine ne sont guère repris dans d'autres traités du XVIe siècle, où les passages évangéliques sont privilégiés. C’est la raison pour laquelle le dossier ne porte que sur l'exégèse de ces passages. À savoir :

- Mt 26, 69-75 par. : le reniement de Pierre ;

- Lc 15 par. : les eux paraboles de la brebis et de la drachmes perdues et l'histoire dite du fils prodigue ;

- Mt 10, 33 par. : Jésus affirmant que celui qui le reniera sera renié par Dieu.

L'étude porte donc sur les commentaires que proposent de ces passages un certain nombre d'exégètes. Le corpus (voir Annexe) comprend des ouvrages qui n'ont pas tous les même statut, et il n'est pas homogène, mais il semble que, tel quel, il est susceptible de fournir une idée de la production exégétique au long du XVIe siècle.

L'exemple du reniement de Pierre dans la première moitié du siècle : dans la continuité de la tradition médiévale

D’emblée, les lectures conduisent à distinguer deux périodes. On croit en effet percevoir que jusqu'à la mi-XVIe siècle, l'exégèse des passages retenus est bien inscrite dans une tradition issue du Moyen Âge et qui pourrait être assez volontiers qualifiée de « polymorphe ». 
Cette tradition, dont rendent compte la Glose ordinaire, la Catena aurea, recueil de citations patristiques sur les textes évangéliques élaboré par Thomas d’Aquin, ainsi que le commentaire de ce dernier sur l’Évangile selon Matthieu, se retrouve diversement mise en œuvre certes, chez des auteurs comme Érasme, Lefèvre d’Étaples, Martin Bucer, Luther ou le dominicain Cajetan. Il serait toutefois faux de dire que ces auteurs proposent des interprétations identiques des trois passages considérés : c’est même tout le contraire qui se produit. Par exemple, le reniement de Pierre, depuis Ambroise de Milan qui insistait sur la valeur à conférer aux larmes de Pierre ${ }^{6}$, peut prendre valeur de texte de conversion :

\footnotetext{
«Bonnes larmes, qui lavent la faute. En somme, ceux sur lesquels Jésus se retourne pour les regarder, ils sanglotent. Une première fois, Pierre a renié, et il n’a pas pleuré ; parce que le Seigneur ne s'était pas retourné pour le regarder. Une deuxième fois, il a renié, mais il n’a pas pleuré ; parce que jusque-là le Seigneur ne s’était pas retourné pour le regarder. Il a renié aussi une troisième fois, mais Jésus s'est retourné pour le regarder, et notre homme a pleuré très amèrement. Retournetoi pour nous regarder, Jésus, afin que nous sachions pleurer abondamment sur nos péchés et laver notre faute. De là, même la chute des saints est utile : il ne m’a nui en rien que Pierre renie, il m’a profité qu’il se corrige. »
}

Cette interprétation est connue au Moyen Âge et donc à l'époque moderne. Cela s'explique aisément, dans la mesure où elle est transmise aussi bien par la Catena aurea ${ }^{7}$ que par la liturgie $^{8}$;

${ }^{6}$ Ambroise, Expositio sur l'Évangile selon Luc, dans Patrologie latine de Migne, vol. XV, col. 1826, nº89 : Bonce lacryme, quce lavant culpam. Denique quos Jesus respicit, plorant. Negavit primo Petrus, et non flevit ; quia non respexerat Dominus. Negavit secundo, non flevit; quia adhuc non respexerat Dominus. Negavit et tertio, respexit Jesus, et ille amarissime flevit. Respice, Domine Jesu, ut sciamus nostrum deflere peccatum, lavare delictum. Unde etiam lapsus sanctorum utilis : nihil mihi nocuit quod negavit Petrus, profuit qod emendavit

7 P. A. Guarenti (éd.), S. Thomce Aquinitatis Catena aurea in quatuor evangelia, Taurini et Rome, 1953, 2 vol., t. II, p. 296-297 : c'est un autre passage d'Ambroise, au sens identique, qu'a retenu Thomas. Quare flevit ? Quia erravit ut homo : lacrymas ejus lego, satisfactionem non lego. Lavant lacrymce delictum, quod voce pudor est confiteri. Negavit primo et secundo, et non flevit, quia adhuc non respexerat Dominus ; negavit tertio, respexit eum Jesus, et amarissime flevit. Ettu si veniam vis mereri, dilue lacrymis culpam tuam. « Pourquoi a-t-il pleuré ? Parce qu'il s'est trompé, en tant qu'homme. Les larmes lavent la faute, qu’on a honte de reconnaître en paroles. Il a renié une première et une deuxième fois, et il n’a pas pleuré ; parce que le Seigneur jusque-là ne s'était pas retourné pour le regarder ; il a renié une troisième fois, Jésus s'est retourné pour le regarder, et il a pleuré très amèrement. Et toi, si tu veux mériter le pardon, dissous ta faute dans les larmes. »

${ }^{8}$ L'offices des laudes des dimanches I et II comporte en effet une hymne latine due à Ambroise dont la cinquième strophe est précisément :

Jesu, labantes respice

Et nos videndo corrige ;

Si respicis, lapsus cadunt

Fletuque culpa solvitur.

Voir Les Hymnes de Liturgia horarum, texte latin et traduction liturgique en langue française, Paris, 1990, p. 32. 
c'est ainsi qu'on la retrouve notamment chez Érasme qui explique l'emploi dans la Vulgate du mot impetus $^{9}$ :

« Par ce terme, donc, Marc cherche à exprimer le changement violent et soudain de Pierre. »

L'accent est bien mis sur le repentir, la mutatio, le changement, la conversion, ce qui est également le cas, mais avec moins de force, chez le dominicain Cajetan, en $1540^{10}$ :

« [Jésus] est décrit comme se tournant [conversus], puisque jusque-là il s’était détourné de Pierre [aversus], le privant de la grâce. »

L'accent ne paraît ici mis que sur le rôle de la grâce dans la conversion de Pierre ; l'ide est-elle exactement la même, lorsque Cajetan écrit ${ }^{11}$ :

« Pierre a manqué [de l’aide de Dieu] [...] à cause de cela il est tombé et a perdu la grâce de la charité. De même Moïse ou David sont tombés. »

On se rapproche assurément de l'interprétation qui insiste davantage sur la faute de Pierre et la solitude absolue de Jésus qui en résulte, sur quoi insiste Jacques Lefèvre d’Étaples ${ }^{12}$.

De même, la parabole de la brebis perdue connaît des éclairages quelque peu différents, mais on retrouve l'étendue de cette palette dans la tradition médiévale. Dans cette première moitié du XVIe siècle, il semble bien que les passages retenus n’ont suscité aucune relecture significative.

Dire la "Grande Cassure » dans la seconde moitié du siècle : les exemples de la brebis perdue et du fils prodigue

Si l'on s'intéresse maintenant aux commentaires de la parabole de la brebis perdue et de l'histoire dite du fils prodigue, on constate avec la seconde moitié du XVIe siècle un changement important : les exégètes trouvent alors dans la tradition médiévale, « polymorphe », de quoi dire la " grande cassure » confessionnelle

${ }^{9}$ Desiderii Erasmi Roterodami Opera omnia..., Leyde, 1705, t. VI, Novum Testamentum..., col. 209, n. 49 : Hoc igitur verbo Marcus exprimere studuit vehementem ac subitum Petri mutationem.

10 Thomas de Vio, dit Cajetan, ... in quatuor Evangelia [...] ad sensum quem vocant litteralem commentarii, Paris, 1540, fol. $309 v^{\circ}$ : Conversusque describitur quoniam tunquam aversus a Petro substractione gratice fuerat.

${ }^{11}$ Ibid., fol. $146 \mathrm{r}^{\circ}$ : Petrus caruit [Dei auxilio] [...] ideo cecidit et perdidit auxilio charitatis gratiam. Sic Moyses sic David cecidit?

${ }^{12}$ Michael Screech (éd.), Jacques Lefèvre d'Étaples, Le Nouveau Testament, Paris et La Haye, 1970, 2 vol. 
Le premier chez qui l'on repère une telle utilisation est Jean Calvin, dans son Harmonie évangélique ${ }^{13}$. Cela n’a rien d'étonnant: Calvin est à coup sûr l'initiateur du processus de «construction confessionnelle» dans l'espace français, et le premier à avoir vu pleinement les conséquences ecclésiologiques de sa théologie ${ }^{14}$.

Il n’est pas seul : la production exégétique postérieure consultée, les Homélies sur la parabole de l'enfant prodigue du docteur de la faculté de théologie de Paris, Claude d’Espence (Paris, s.d.), la Bibliotheca sancta de Sixte de Sienne (Lyon, 1592), les commentaires des quatre évangiles du jésuite Jean Maldonnat (Pont-à-Mousson, 1596) et les Annotationes de Théodore de Bèze, le successeur de Calvin à Genève (Genève, 1594), présentent le même phénomène, sans qu’on puisse jamais distinguer des discours confessionnels rivaux.

Ainsi, à propos de la brebis perdue, dans la première moitié du XVIe siècle, on reprend l’interprétation traditionnelle, ainsi exprimée dans la Glose ordinaire ${ }^{15}$ :

« [Mt 18] Le Seigneur eut cent brebis, à la nature douée de raison, les anges et les hommes, qui demeurèrent dans sa perfection. Mais l'une a erré, de même que l’homme a péché.

[Lc 15] Cent brebis - parce que le nombre cent est parfait, Dieu eut cent brebis, nombre parfait, quand il a créé les anges et la substance des hommes. Mais l'une s’est perdue, de même que l’homme a péché. »

On peut par ailleurs lire ce passage de Grégoire le Grand, dans la Catena aurea de Thomas d'Aquin $^{16}$ :

« Une brebis alors s'est perdue de même que l’homme en péchant a laissé la pâture de la vie. Mais dans le désert quatrevingt-dix-neuf étaient demeurées : parce que le nombre des créatures douées de raison, bien sûr les anges et les hommes nombre fait pour ressembler à Dieu -, l'homme parti, était diminué ; d'où il s'ensuit Ne laisse-t-il pas les quatre-vingt-dixneuf dans le désert ? parce que bien sûr il laisse les chœurs des Anges au Ciel. Alors l'homme abandonne le Ciel, parce qu'il a péché [...]. »

${ }^{13}$ Commentaires de Jehan Calvin sur le Nouveau Testament, Paris, 1852, Toulouse, 1892-1894, p. $185-319$.

${ }^{14}$ Olivier Fatio, « Introduction », dan Jean Calvin, Le traité des scandales, Genève, 1984, p. 12.

15 Glosa ordinaria in quatuor evangelia, Strasbourg, c.1481, incunable conservé à la Bibliothèque du Saulchoir à Paris (Rés. Inc. A6) : [Mt 18] Dominus centum oves hubuit [sic pour habuit] cum rationali natura, angelos et hominem, qui in sua perfectione mansuerunt. Sed una eravit, cum homo peccavit. [Lc 15] Centum oves - quia centenarius numerus perfectus est, deus centum oves, perfectum numerum habuit, cum angelos et hominum substantiam creavit. Sed una peribit, cum homo peccavit.

${ }^{16}$ Catena aurea, ouvr. cit., t. II, p. 213 : Una ovis tunc periit quando peccando homo pascua vitce reliquit. In deserto autem nonagintanovem remanserant : quia rationatum creaturarum numerus, Angelorum videlicet et hominum, qui ad videndum Deum conditus fuerat, pereunte homine, erat imminutus ; unde sequitur Nonne dimittit nonaginta novem in deserto ? qui scilicet Angelorum choros reliquit in cœlo. Tunc autem homo deseruit, cum peccavit. [...] 
Les quatre-vingt-dix-neuf brebis sont les anges, la centième, celle qui se perd, l’humanité déchue ; Jacques Lefèvre d’Étaples, prêchant dans le diocèse de Meaux vers 1520, n’explique rien d'autre à ses ouailles ${ }^{17}$ :

« La brebis errante, c’est nous, c’est le genre humain. »

Et Cajetan, vingt ans plus tard, estime qu’il n’est pas difficile de décrypter la parabole ${ }^{18}$ :

« La parabole est suffisamment claire. Et elle signifie que c’est Jésus lui-même, fils de Dieu, qui a cent brebis (c'est-àdire tous les élus), parmi lesquelles celle qui est perdue est la nature humaine. Les quatre-vingt-dix-neuf brebis laissées au désert sont tous les anges élus, auxquels il manque l'espèce humaine pour accomplir le nombre des élus. Le désert est le ciel, séparé absolument de nous. L'arrivée pour rechercher la brebis perdue est l'incarnation du fils de Dieu. La mise de la brebis sur l'épaule est la rédemption de l'espèce humaine. Et cela parce qu'il l’a fait spontanément, de sorte qu'il est décrit comme joyeux. Le retour vers la maison est la résurrection et l'ascension au Ciel. La convocation des amis et des voisins est l'association des anges qui se réjouissent du salut des hommes. »

On ne saurait être plus clair, en effet ; et Cajetan, au même passage, à propos cette fois de la drachme perdue, propose la même interprétation ${ }^{19}$ :

« Dix drachmes, neuf sont les ordres des anges, et la nature humaine, qui ont l'image de Dieu de même que les pièges de monnaie qui sont appelées des drachmes ont l'image du prince. La drachme perdue est la nature humaine [...]. »

La parabole est bien alors une manière d'exprimer toute l'histoire du salut de l’humanité après la Chute. Jean Calvin cependant explique différemment le début de Lc $15^{20}$ :

«Ainsi que saint Luc couche son récit, il regarde une fin un peu diverse [par rapport au but qu'assigne à la parabole Matthieu] : assavoir d'autant que tout le genre humain est à Dieu, qu'il faut rassembler ceux qui sont éloignés de lui, et que quand ceux qui vivaient ès [=en les] idolâtries viennent à s'amender, il s'en faut réjouir ni plus ni moins qu'un homme qui recouvrera sans y penser une chose dont la perte l'avait fort ennuyé. »

${ }^{17}$ Michael Screech (éd.), Jacques Lefèvre d'Étaples, Epistreset Evangiles pour les cinquante et deux sepmaines de l'an, fac-similé de l'éd. de Paris, 1523, Genève, 1964, fol. CLXXXIIIrº.

${ }^{18}$ Thomas de Vio, dit Cajetan, ouvr. cit., fol. $276 v^{\circ}$ : Parabola satis clara est. Et significat ipsum Jesum filium dei habentem centum oves (hoc est universos electos), quarum una perdita, natura est humana : nonaginta vero novem dimissce in deserto, omnes sunt electi angeli, quibus deest ad perficiendum electorum numerum genus humanum. Desertum autem, cœlum est separatum omnino a nobis. Adventus ad qucrendum ovem perditam incarnatio est filii dei. Impositio ovis in humero redemptio est humani generis. Et hoc quia sponte fecit, ideo gaudens describitur. Reditus domum, ressurrectio et ascensio est in colum. Convocatio amicorum et vicinorum, associatio est angelica gaudens de reparatione humana.

${ }^{19}$ Ibid. : Decem drachme, novem sunt ordines angelorum, et humana natura, imaginem habentes dei, sicut numismata quce drachmee appellantur imaginem habent principis. Drachma perdita est humana natura [...].

${ }^{20}$ Jean Calvin, ouvr. cit., p. 465. 
Comme Calvin, Théodore de Bèze propose de voir dans les quatre-vingt-dix-neuf brebis une image des justes, et de la centième une image de celui qui se détourne de Dieu, le pécheur qui, finalement, va se repentir ${ }^{21}$. Le jésuite Maldonnat en vient même à déclarer ${ }^{22}$ :

« À ce propos, il y a un grand débat entre les commentateurs, pour savoir qui sont les quatre-vingt-dix-neuf brebis ; les auteurs très anciens disaient que celles qui n’erraient pas étaient les bons anges. En effet, les brebis étaient toutes les créatures de Dieu douées de raison, parmi lesquelles les unes, c'est-à-dire les hommes, ont erré ; les autres, c'est-à-dire les anges, n'ont pas erré. [...] Mais il n’est pas douteux que le Christ n’ait parlé que des seuls hommes [...]. »

Le refus de l'interprétation traditionnelle, encore exprimée par Cajetan, on l'a vu, est ici très net : s'il admet, d'abord, que la question est débattue, Maldonnat la tranche très vite, dans un sens vers lequel le Réformateur de Strasbourg Martin Bucer, quarante ans plus tôt, ne pouvait se tourner ; se demandant qui étaient les quatre-vingt-dix-neuf brebis, dès lors qu’on admettait que la centième était les hommes, ce dernier refusait en effet même d'envisager la question ${ }^{23}$ :

« Nous ne discuterons donc pas pour savoir qui sont ces justes qui n’ont pas besoin de pænitentia, parce que tous les hommes sont pécheurs, et perpétuellement repentants. »

Et il le faisait d'une manière bien luthérienne : semper peccator, semper penitens... ${ }^{24}$. C'est chez Calvin, dans le passage cité, que le processus de « construction confessionnelle » paraît enfin pris en compte, à travers une interprétation jusque-là peu fréquente : le pécheur devient le fidèle de l’autre confession, stigmatisée par Calvin comme « idolâtrique ». Ainsi, Calvin paraît interpréter Lc 15, 3-10 non seulement comme un récit de conversion du pécheur, mais même comme un récit de conversion au protestantisme réformé. On s'avance ici vers ce qui pourrait être une véritable relecture du texte évangélique, dans la mesure où il semble bien que le Réformateur de Genève fasse parler ce texte de la situation confessionnelle nouvelle.

L'histoire dite du fils prodigue suscite chez ses commentateurs le même type de discussion : comment faut-il interpréter les figures du fils cadet, le prodigue, et du fils aîné ? Représente-elles

${ }^{21}$ Théodore de Bèze, ouvr. cit., p. 107 sur Mt 18.

22 Jean Maldonnat, ouvr. cit., col. 732 : De eo autem inter interpretes magna discepatio est, quce illce 99. sint oves, quxe non erraverunt, vetustissimi auctores angelos bonos dixerunt esse. Oves enim Dei esse omnes creaturas rationales, ex quibus alice id est, homines erraverint ; alice, id est, angeli, non erraverint. [...] Sed dubium non est, quin de solis hominibus Christus loquatur [...].

${ }^{23}$ Martin Bucer, ... In quatuor Evangelia [...] ad sensum quem vocant litteralem commentarii, Paris, 1540, fol. $144 \mathrm{r}^{\circ}$ : Non igitur disputemus qui justi sint, pænitentia non egentes, quum universi peccatores sint, eoque continuo resipiscentes $[\ldots]$.

${ }^{24}$ Henri Strohl, Luther jusqu'en 1520, Paris, $2^{\text {nde }}$ éd., 1962, p. 119. 
respectivement l'humanité et les anges ? Déjà Ambroise mettait en doute cette interprétation, dans un passage qui, une fois encore, est dans la Catena aurea $^{25}$ :

« Il y en a qui disent des deux fils que l'aîné est les anges, et le cadet l’homme, parce qu'il a fait un long voyage quand il est tombé du ciel et du paradis sur la terre [...]. Mais ce sens semble certes pieux, pourtant je ne sais s'il est vrai : parce que le plus jeune fils vient spontanément au repentir [...]; alors que le Seigneur appelle l'espèce humaine à venir au repentir, cependant que celle-ci ne pense pas à repartir d'où elle était tombée. Ensuite le fils plus âgé s'attriste du retour et du salut de son frère ; alors que le Seigneur dit qu'il y a liesse chez les anges quand un pécheur se repent. »

Cette interprétation n’est plus mentionnée par Claude d’Espence ${ }^{26}$ :

«Ses deux enfants sont ou l'Ange et l'homme, ou le Juif et le Gentil, comme deux souches du genre humain ; ou mieux généralement justes et innocents, c’est-à-dire qui tel s’estime et pense, et le pécheur qui tel aussi s’avoue être et confesse. »

L’interprétation générale est bien celle qu'exprime en ces termes Cajetan ${ }^{27}$ :

« Cette parabole est claire en soi [...]. Donc l’homme est Dieu. Les deux fils sont l'espèce des justes qui persévèrent dans toute leur vie sans péché mortel, et le genre des pécheur (toutefois se repentant). »

Il y aurait d’un côté les justes, de l'autre les pécheur ; la fin de la parabole conduit toutefois ces commentateurs à nuancer les choses. Jean Maldonnat expose la discussion dans son entier : il refuse, en invoquant Tertullien, de penser que, comme on l'a dit, les deux fils puissent être les deux « peuples », juif et païen ; faut-il alors y voir l'opposition entre publicains et pharisiens, ou bien entre hommes et anges, ou encore quelque figure des deux larrons de la Crucifixion ? Il en arrive à une conclusion $^{28}$ :

${ }^{25}$ Catena aurea, ouvr. cit. : Sunt qui dicant de duobus filiis istis seniorem Angelos esse, juniorem vero hominem, qui in longinquam peregrinationem abjerit, quando in terram de cœelis et paradiso cecidit [...]. Sed hic senss pius quidem videtur, nescio tamen si verus sit : quia junior filius ad pœenitentiam venit sua sponte [...] ; Dominus autem veniens ad pœnitentiam vocavit humanum genus, dum sponte sua regredi, unde ceciderat, non cogitaret. Deinde senior filius in reditu et salute fratris sui tristatur ; cum Dominus dicat lcetitiam esse apud angelos super uno peccatore penitentiam agente.

${ }^{26}$ Claude d'Espence, ouvr. cit., fol. $2 \mathrm{r}^{\circ}$.

${ }^{27}$ Cajetan, ouvr. cit., fol. $277 \mathrm{r}^{\circ}$ : Parabola hrec in se clara est [...]. Homo igitur quidam deus est. Duo filii genus justorum perseverantium in tota vita sine peccato mortali et genus lapsorum (pœenitentium tamen) sunt.

${ }^{28}$ Jean Maldonnat, ouvr. cit., col. 259-260 : Non ergo dubito per majorem natu filium omnes justi, per minorem omnes peccatores intelligi, sive Judœi ante passum Christum ; sive postea Christiani sint. Num qui in Ecclesia Dei sunt, filii Dei sunt, gentiles non filii, non domestico sunt Dei [...].

Igitur filii Dei dicuntur non solum justi, quos propria quadam ratione scriptura filios solet appellare; sed etiam peccatores, qui fidem habent, quia fides etiam nos suo quodam modo filios Dei facit; sed filios mortuos, filios perditos, sicut fides ipsa sine charitale vera quidem fides, sed mortua tamen est. Ergo major natu filius est verus christianus, minor non verus est. 
« Je ne doute donc pas qu’à travers le fils aîné soient entendus tous les justes, et à travers le cadet tous les pécheurs, ou les juifs avant la passion du Christ, ou ensuite les chrétiens. En effet, ceux qui sont dans l’Église de Dieu sont les fils de Dieu, les païens ne sont pas les fils ni les domestiques de Dieu [...].

Donc on dit fils de Dieu non seulement les justes, que pour quelque raison appropriée l’Écriture a accoutumé d’appeler fils ; mais même les pécheurs, qui ont la foi, parce que la loi nous fait fils de Dieu à sa manière ; mais ce sont des fils morts, des fils perdus, de même que la foi elle-même sans charité est certes la vraie foi, mais est cependant morte. Donc le fils aîné est le vrai chrétien, le cadet n’est pas vrai chrétien. »

La discussion sur le fait que les pécheurs méritent eux aussi le titre de «fils de Dieu », dans la mesure où elle se conclut sur cette distinction entre vrai et faux chrétiens parait trouver sa justification dans le contexte de la «construction confessionnelle». Le commentaire oublie la fin du récit évangélique, qui faisait apparaître l'aîné comme la figure du superbus, qui s’enorgueillit de sa fidélité et de sa constance, et ne met plus en valeur la jalousie de ce dernier, par opposition au repentir du cadet, comme si désormais il convenait surtout de dire la situation ecclésiologique nouvelle - et de la conforter par la valorisation justement de la fidélité et de la constance confessionnelles.

La question demeure, pourtant, de savoir si on là à proprement parler un phénomène de relecture, au sens donné à ce terme par les exégètes contemporains. Or, à strictement parler, il ne saurait s'agir d'une relecture, dans la mesure où les interprétations mises désormais en avant se trouvaient déjà aux époques antérieures. À propos de la Brebis perdue, la Glose ordinaire explique ${ }^{29}$ :

«Celle qui est perdue, ce sont tous les pécheurs qui, à travers les pénitences, sont réconciliés à Dieu. »

Quant à la Catena aurea, elle présente ce passage de Jérôme qui va dans le même sens ${ }^{30}$ :

\footnotetext{
« D’autres pensent que par les quatre-vingt-dix-neuf brebis est compris le nombre des justes, et dans la brebis unique le nombre des pécheurs, selon ce que dans un autre passage il avait dit : Je ne suis pas venu pour appeler les justes, mais les pécheurs. »
}

L'explication existe donc déjà au Moyen Âge, même si on n’y insiste pas. La tradition médiévale n'est-elle pas suffisamment riche pour qu'on y trouve de quoi mettre en valeur ce sur quoi on veut insister ? Cela expliquerait-t-il q'il n’y ait pas eu, en conséquence, au long du XVIe siècle de réinterprétation, mais plutôt nouvelle orientation donnée à la lecture des passages retenus ? On serait tenté de le croire. Encore faut-il s'attarder un instant sur le devenir de l'exégèse de l'épisode du reniement de Pierre.

Une relecture aux XVIe et XVIIe siècles: le reniement de Pierre chez Jean Calvin et dans les Provinciales

${ }^{29}$ Ouvr. cit. : Quis perdita, omnes peccatores qui per pœnitentias reconciliantur deo.

${ }^{30}$ Catena aurea, ouvr. cit., t. I, p. 271 : Alii vero nonagintanovem ovibus, justorum putant numerum intelligi, et in una ovicula peccatorum secundum quod in alio loco dixerat : Non veni vocare justos, sed peccatores. 
Ni Maldonnat ni Sixte de Sienne ne commentent de manière originale l'épisode du reniement de Pierre. Il en va autrement de Jean Calvin. Celui-ci commence ainsi son commentaire ${ }^{31}$ :

\begin{abstract}
« La chute de Pierre qui nous est ici récitée est un fort beau miroir de notre infirmité. Puis après en sa repentance nous est proposé un exemple singulier de la bonté et miséricorde de Dieu. Ainsi cette histoire qui est racontée touchant un homme seul, contient une doctrine commune à toute l'Église, et fort utile : tant pour avertir ceux qui sont debout de se tenir en crainte et sollicitude, comme pour redresser et consoler ceux qui sont chus, en leur proposant espérance de pardon. Et premièrement il faut noter que Pierre a fait inconsidérément quand il est entré jusques en la salle du Grand Sacrificateur. Il est vrai que c'était bien un acte appartenant à son devoir, de suivre son Maître ; mais vu qu'il avait été averti de son révoltement et renoncement, il devait plutôt e tenir en quelque coin, pour éviter toute occasion de pécher. »
\end{abstract}

Calvin ne paraît pas parler ici de ce que les autres commentateurs du passage mettent en lumière. Le vocabulaire employé (chute de Pierre, infirmité, repentance, ceux qui sont chus, révoltement, renoncement) renvoie en effet directement aux apostasies telles que les milieux réformés, en particulier genevois, avaient l'habitude de les décrire ; et le conseil de ne pas se mettre en situation de péché et de chercher plutôt en cas de tentation de se retirer " en quelque coin » est celui que C alvin donne aux Français gagnés à « l’Évangile » et menacés de persécution ! Il faut rejoindre les Églises de réfugiés où on pourra être vraiment «fidèle ». L'exégèse calvinienne use ici de mots nouveaux pour évoquer la situation présente : ne serait-on pas en droit de parler alors d'une véritable relecture ?

Le cas est peut-être isolé au XVIe siècle ; le même épisode évangélique fournit cependant au XVIIe un autre exemple de relecture. On est même étonné de ne pas l'avoir rencontré plus tôt dans le temps. Comment en effet s'expliquer que commenter le reniement de Pierre n'ait pas fourni l'occasion aux exégètes du XVIe siècle de débattre du problème de la grâce et des œuvres ? On a certes vu que Cajetan insiste surtout sur le fait que Pierre a manqué de l'aide de Dieu, ce qui explique sa faute ; mais le dominicain n’en profite pas pour entrer dans la polémique de la grâce et des œuvres. Ce n’est que dans les Provinciales de Pascal, à la mi-XVIIe siècle, que l'exégèse en saisit l’occasion.

Dans la troisième des Provinciales, datée du 9 février 1656, en effet, Pascal prend la défense d'Antoine Arnauld. Celui-ci vint de voir sa seconde Lettre à un duc et pair censurée par la faculté de théologie de Paris (31 janvier 1656) ; on lui reprochait la manière dont il y avait commenté l'épisode du reniement de Pierre : le récit « nous montre un juste en la personne de saint Pierre, à qui la grâce, sans laquelle on ne peut rien, a manqué ». Pascal laisse entendre qu'on l'a de cette manière accusé de « calvinisme ». Citant consciencieusement la Défense d’Arnauld, Pascal conduit ses lecteurs à douter qu’une telle proposition soit réellement hérétique ${ }^{32}$ :

« [M. Arnauld] fait voir que saint Augustin dit, en un endroit qu'il cite : Que Jésus-Christ nous montre un juste en la personne de saint Pierre, qui nous instruit par sa chute de fuir la présomption. Il en rapporte un autre du même Père, qui dit : Que Dieu, pour montrer que sans la grâce on ne peut rien, a laissé saint Pierre sans grâce. Il en donne un autre de saint Chrysostome, qui dit : Que la chute de saint Pierre n'arriva pas pour avoir été froid envers Jésus-Christ, mais parce que la

\footnotetext{
31 Jean Calvin, ouvr. cit., p. 683.

${ }^{32}$ Blaise Pascal, Les Provinciales, éd. par Antoine Adam, Paris, 1981, p. 55-56.
} 
grâce lui manqua ; et qu'elle n'arriva pas tant par sa négligence que par l'abandon de Dieu, pour apprendre à toute l'Église que sans Dieu l'on ne peut rien. Ensuite de quoi, il rapporte sa proposition accusée, qui est celle-ci : Les Pères nous montrent un juste en la personne de saint Pierre, à qui la grâce, sans laquelle on ne peut rien, a manqué.

C'est sur cela qu'on essaie en vain de remarquer comment il se peut faire que l'expression de M. Arnauld soit autant différente de celles des Pères que la vérité l'est de l'erreur, et la foi de l'hérésie : car où en pourrait-on trouverla différence ? Serait-ce en ce qu'il dit : Que les Pères nous montrent un juste en la personne de saint Pierre ? Saint Augustin l'a dit en ces mots propres. Est-ce en ce qu'il dit : Que la grâce lui a montré ? Mais le même saint Augustin qui dit, que saint Pierre était juste, dit qu'il n'avait pas eu la grâce en cette rencontre. Est-ce en ce qu'il a dit: Que sans la grâce on ne peut rien ? Mais n'est-ce pas ce que saint Augustin dit au même endroit, et ce que saint Chrysostome même avait dit avant lui, avec cette seule différence qu'il l'exprime d'une manière bien plus forte, comme en ce qu'il dit : Que sa chute n'arriva pas par sa froideur, ni par sa négligence, mais par le défaut de la grâce, et par l'abandon de Dieu ? »

On n’a vraiment affaire là qu’à quelque « hérésie imperceptible ${ }^{33}$ », pur prétexte pour les jésuites de s’en prendre aux prétendus jansénistes.

On est étonné : la querelle janséniste a u susciter un phénomène de relecture de ce passage, alors que la « construction confessionnelle », au cœur de laquelle le débat sur la grâce passe pour avoir été prégnant, ne l'avait pas fait. Ce constat remet en question la validité du corpus choisi : les textes scripturaires retenus ne se seraient pas prêtés, en fait, à relectures au XVIe siècle. Mais pourquoi ? Une explication pourrait être avancée, quoique encore seulement à titre d'hypothèse: dans l'espace français tout au moins, principalement représenté par le corpus étudié, la rupture confessionnelle a peut-être moins porté sur la grâce que sur l'eucharistie. Les prétendus jansénistes s'opposent avec vigueur aux réformés précisément sur ce terrain ; et ils s’estiment en droit d'être bons catholiques tout en étant, sur la grâce, « vrais disciples de saint Augustin ». Mais la frontière confessionnelle s'étant linéarisée, leurs adversaires catholiques dénoncent en fait en eux des « crypto-calvinistes ».

\footnotetext{
${ }^{33}$ Ibid., p. 57.
} 